\title{
Is magnetic resonance safe in implanted cardiac devices patients?
}

\author{
Giulia Cernuschi • Maurizio Cringoli • \\ Gruppo di Autoformazione Metodologica (GrAM)
}

Received: 18 January 2012 / Accepted: 21 March 2012/Published online: 11 April 2012

(C) SIMI 2012

\section{Background}

Magnetic resonance imaging (MRI) is often avoided in patients with implanted cardiac devices such as pacemakers (PMs) and implantable cardioverter-defibrillators (ICDs) because of safety concerns [1]. The overwhelming majority of implanted devices are considered a contraindication to MRI by the U.S. Food and Drug Administration and by devices manufacturers [2]. Exposure to electromagnetic fields could cause variations in sensing, lead impedance and capture threshold. Moreover, it can revert the device to a back-up programming mode known as "power on reset", during which the device is susceptible to inhibition of pacing output and activation of antitachycardia therapies.

These alterations together with interference with the ICDs functions may cause adverse clinical events, such as induction of dysrhythmias.

It is estimated that during their lives, up to $75 \%$ of patients with cardiac devices need an MRI examination. While research is attempting to develop an MRI-compatible pacemaker, many patients will still retain the old one for many years.

\section{Summary}

Nazarian et al. [1] evaluate in a prospective non-randomized trial the safety of a $1.5 \mathrm{~T}$ MRI protocol for patients with implanted cardiac devices (ICD and PM). 438 participants

G. Cernuschi $(\bowtie) \cdot$ M. Cringoli

Medicina Interna II, Ospedale L. Sacco, Universita ‘ degli Studi di Milano, Via G.B. Grassi, 74, 20157 Milan, MI, Italy

e-mail: giulia.cernuschi@studenti.unimi.it
(54\% with PM and $46 \%$ with ICD implanted, respectively, after the 1998 and 2000) were enrolled and underwent 555 MRI examinations. Patients with a recent implant ( $<6$ weeks), those with abandoned or epicardiac leads, and PM-dependent patients with an ICD were excluded. According to the experimental protocol, an asynchronous pacing mode (VOO/DOO) was programmed in the pacemaker-dependent patients, while an inhibited pacing mode (VVI/DDI) was used for the other patients. The ICD function of non-PM-dependent patients was disabled. During the MRI examination, blood pressure, electrocardiography, pulse oximetry and symptoms were monitored by a nurse with experience in cardiac life support and device programming who had an immediate backup from an electrophysiologist. Device variables including sensing, impedance, capture threshold and battery voltage were evaluated before MRI examination, immediately after MRI and after 3-6 months. Variations exceeding 30, 40 and $50 \%$ for, respectively, lead impedance, sensing and capture threshold were considered as significant changes in lead performance.

Baseline and immediate follow-up interrogations were performed in all 438. Long-term follow-up device variables were available for 266 patients $(61 \%) .3$ of 438 patients $(0.7 \%)$ experienced acute power-on-reset events. None of them had device dysfunction during long-term follow-up (3-6 months). Right ventricular sensing and atrial, right and left ventricular impedances were reduced immediately after MRI. At long-term follow-up in $61 \%$ of the patients, decreased right ventricular (RV) sensing and lead impedance, increased RV capture threshold and decreased battery voltage were noted. The observed changes did not require device revision or reprogramming. The distributions of changes in device variables were within the $20 \%$ at baseline for most participants. Thoracic MRI sequences had a greater effect on device variables and were more 
likely to result in artifacts (for instance, image distortion, signal voids or bright areas and poor fat suppression).

\section{Strengths of the study}

- The issue of the study was clinically relevant;

- A long term follow-up was performed;

- Although not all available cardiac devices could be used, a table with all the technical details of the ones tested was provided in the paper;

- The protocol adopted was clear and reproducible.

\section{Weakness of the study}

- The study was not a controlled trial;

- The follow-up was not completed; 3-6 months followup information about the devices' variables were collected directly in only $61 \%$ (266) of the patients, clinical information regarding the other 172 patients was just obtained by telephone or with an interview, but was missing for $43(10 \%)$ of the patients. Moreover it is not known if device-related malfunctions or dysrhythmias occurred in these patients, most of whom were at higher risk of events (thoracic MRI).

\section{Question marks}

- The absence of identified adverse events is not equivalent to demonstrating the absolute safety of MRI in patients with cardiac devices. In the medical literature, some deaths putatively associated with MRI examination in patients with pacemakers have been reported [3, 4]. None of them occurred during appropriate physician-supervised monitoring as is the clinical trial setting;
- The study protocol was carried out in a highly qualified health center. It is questionable whether the study protocol can be applied in small centers;

- It is not clear why at least two patients who should not have been included according to exclusion criteria (one PM implanted before 1998 and one ICD implanted before 2000) were included in the study.

\section{Clinical bottom line}

With appropriate safety measures and a proper protocol, MRI examinations can be performed safely in patients with selected cardiac devices. Since changes in device variables and programming may occur, electrophysiologic monitoring and strict follow-up are essential. Extrathoracic MRIs seem to be less dangerous than examination involving the body region near the heart.

Acknowledgments The study was funded by the National Institute of Health and the PJ Schafer Memorial Research Award, which however had no role in the detection and processing of data.

Conflict of interest None.

\section{References}

1. Nazarian S, Hansford R, Roguin A, Goldsher D, Zviman MM, Lardo AC, Caffo BS, Frick KD, Kraut MA, Kamel IR, Calkins H, Berger RD, Bluemke DA, Halperin HR (2011) A prospective evaluation of a protocol for magnetic resonance imaging of patients with implanted cardiac devices. Ann Intern Med 155:415-424

2. Zikria JF, Machnicki S, Rhim E, Bhatti T, Graham RE (2011) MRI of patients with cardiac pacemakers: a review of the medical literature. AJR Am J Roentgenol 196:390-401

3. Irnich W, Irnich B, Bartsch C, Stertmann WA, Gufler H, Weiler G (2005) Do we need pacemakers resistant to magnetic resonance imaging? Europace 7:353-365

4. Avery JK (1988) Loss prevention case of the month: not my responsibility! J Tenn Med Assoc 81:523 